\title{
COVID-19 vaccine hesitancy and anti-vaxxers - supporting healthcare workers to navigate the unvaccinated: Reflections from clinical practice
}

\author{
J Hoare, ${ }^{1,2} \mathrm{MB} \mathrm{ChB}, \mathrm{PhD}$ (D); M Mendelson, ${ }^{3,4} \mathrm{MBBS}, \mathrm{PhD} ;$ L Frenkel, ${ }^{1}$ MA (Clin Psychol) \\ ${ }^{1}$ Department of Psychiatry and Mental Health, Faculty of Health Sciences, University of Cape Town, South Africa \\ ${ }^{2}$ Peninsula Medical School, Faculty of Health, University of Plymouth, UK \\ ${ }^{3}$ Division of Infectious Diseases and HIV Medicine, Faculty of Health Sciences, University of Cape Town, South Africa \\ ${ }^{4}$ Department of Medicine, Faculty of Health Sciences, University of Cape Town, South Africa
}

Corresponding author: J Hoare (jackie.hoare@uct.ac.za)

\begin{abstract}
An important step in preparation for the fourth COVID-19 wave is to provide healthcare workers (HCWs) with skills to facilitate behaviour change in vaccine-hesitant patients. Convincing members of the public who are vaccine hesitant rather than anti-vaxxers should be the focus of our efforts. Our experience is that vaccine-hesitant individuals and anti-vaxxers are generally distinct cohorts, with differing reasons for their vaccine reluctance. If we are to truly address hesitancy, we must take time to understand the reasons for an individual's hesitancy. Developing a conceptual framework and skills for HCWs during encounters with unvaccinated individuals will be important not only for shifting some to get vaccinated, but also to manage the complex emotions that HCWs will undoubtedly be forced to confront during the fourth wave.
\end{abstract}

S Afr Med J 2022;112(1):11-13. https://doi.org/10.7196/SAMJ.2022.v112i1.16208

Being able to conceptualise COVID-19 vaccine-hesitant individuals and anti-vaxxers as distinct populations may aid healthcare workers (HCWs) in managing the challenge of engaging patients who have as yet chosen not to be vaccinated. Understanding the reasons individuals provide for their reluctance to be vaccinated against COVID-19 is useful, but a more helpful approach may be to identify the psychological processes that characterise and distinguish vaccine-hesitant individuals from anti-vaxxers. ${ }^{[1]}$ Developing a conceptual framework and skills for HCWs during encounters with unvaccinated individuals will be important not only for shifting some to get vaccinated but also to manage the complex emotions that HCWs will undoubtedly be forced to confront during the fourth wave. We are concerned about the mental health implications for our already exhausted and overstretched HCWs when the burden of the pandemic will rest largely on the shoulders of the unvaccinated in a context of widely available vaccine. Our experience is that vaccinehesitant individuals and anti-vaxxers are generally distinct cohorts, with differing reasons for their vaccine reluctance. Our approach to management of these two sets of people should reflect these differences.

\section{Discussion}

Our experience of anti-vaxxers admitted to the COVID-19 wards at Groote Schuur Hospital (GSH) in Cape Town, South Africa, as well as the anti-vaccine protest staged outside the hospital on 21 August 2021, is that anti-vaxxers have a tendency towards conspiratorial beliefs linked to their negative attitudes towards vaccination. In comparison with vaccine-hesitant individuals, anti-vaxxers are more likely to believe that their way of life is at threat from hierarchical structures and authoritarianism. They displayed a mistrust of authoritative figures, including scientists and the HCWs delivering care to them in hospital. We experienced COVID-19 anti-vaxxers as being more resistant to evidence-based medical management offered by our team, and found that they would frequently bargain for access to alternative therapies and were more likely to refuse intubation and ICU admission if indicated. The distrust that antivaxxers have for those who represent authority and science provokes resistance to messaging from these sources and an entrenchment of their existing anti-establishment or anti-authority beliefs. As a result, they are less likely to consume and trust information from traditional sources and more likely to obtain information from social media channels that have become notorious for promoting misinformation about COVID-19. Some anti-vaxxers openly deny the improvements vaccination programmes have made to public health over centuries and commonly cite concerns about safety.

We observed a relationship between ivermectin use and antivaxxer beliefs in our patients. Many anti-vaxxers we encountered day to day believed that ivermectin was protecting them despite no current scientific evidence supporting its use for the treatment or prevention of COVID-19. On the whole, clinicians, scientists and public health practitioners do not support the use of ivermectin to treat COVID-19, hence the seeming attraction of this drug to antivaxxers. Anti-vaxxers shared a strong belief that they are 'awake' and that there is a big shift happening in the world. COVID-19 anti-vaxxers use the same arguments made in the 19th century, dating back to the smallpox epidemic. ${ }^{[2]}$ The approach has been to play down the threat from the disease, claim that the vaccine causes illness, is ineffective, or both, declare vaccination to be part of a larger conspiracy, and finally, to use alternative authorities that legitimise their arguments. The history of anti-vaccination is part of a wider story of society wrestling with the promises and perils of advancing technology. It is also a story of activist groups that do not so much 
deny science as cherry-pick information and misinformation to build their nests of belief. ${ }^{[2]}$ Fortunately, anti-vaxxers represent a small proportion of individuals we encounter on the wards who have as yet chosen not be vaccinated.

In contrast, many of our patients are simply hesitant, often because of fear of side-effects, or are interested in being vaccinated but have experienced barriers such as location of vaccine sites, money for travel, child care issues or time off work. Some vaccine-hesitant individuals believe in their ability to resist COVID-19 infection with a healthy diet and a positive attitude. Others expressed a desire to wait to be vaccinated until enough of their friends and family had been vaccinated, were complacent, or did not see the need for the vaccine. As with any medical intervention there is the potential for complications, but the difference is that vaccines are given to healthy people, compounding concern over the risk-benefit ratio for the individual. Fears around public safety, misinformation, perceived difficulty in registering online, and far-removed vaccine sites are factors contributing to vaccine hesitancy. Older individuals may not be tech savvy, making registration on the Electronic Vaccine Data System difficult. Stories of infertility or dying after vaccination are being spread in the community, and many individuals who have comorbidities are particularly concerned about the safety of the vaccine. These reasons are distinct from anti-vaxxers' beliefs in conspiracy theories and myths, such as that mRNA vaccines can be inserted into their DNA or have microchips to track and control them. Many people are anxious about introducing a vaccine into their body because of the rapidly evolving and unprecedented nature of COVID-19.

To counter vaccine hesitancy, we need correct, easily digestible information based on the latest research, easy access to vaccination sites, social marketing campaigns, and community champions to encourage vaccine acceptance. Our role as HCWs is that of advocates, to support a sufficient proportion of vaccine-hesitant people to accept the benefits of the vaccine so as to effectively mitigate the epidemic. Convincing members of the public who are vaccine hesitant rather than anti-vaxxers should be the focus of our efforts. A study on COVID-19 vaccine acceptance and hesitancy in low- to middle-income countries ${ }^{[3]}$ supports our experience of vaccinehesitant individuals at GSH. The study showed that the majority of participants would trust HCWs to help them to decide whether to take the COVID-19 vaccine. Although vaccine hesitancy is concerning, we found that we were able to work with vaccine-hesitant patients while they were admitted to our COVID-19 wards. Concerns about vaccine efficacy often seemed to reflect a lack of information about the vaccine. However, vaccine-hesitant individuals rarely cited conspiracy theories about ulterior motives on the part of HCWs. Many vaccine-hesitant patients admitted to our wards expressed the wish to 'wait and see' before getting vaccinated.

At GSH, as elsewhere in the world, many of the COVID-19-related hospitalisations and deaths involve the unvaccinated, those who have feared and chosen to postpone vaccination. The latter half of the third wave ignited feelings of frustration, with HCWs needing to navigate how to engage with patients who are ambivalent about following scientific evidence for COVID-19 prevention. The daily educational encounters with patients and family members can create a challenging environment that cultivates hopelessness in HCWs, who are already exhausted from treating numerous COVID-19 patients and coping with the daily risk to their mental wellbeing. Some, who have tried to advocate for vaccination on social media platforms, have been exposed to abusive, damaging rhetoric, which erodes empathy. Hence, HCW empathy for unvaccinated COVID-19 patients is being sorely tested, and without the two-way bond of trust, HCWs may run out of fuel.

Managing HCWs' feelings of frustration and exhaustion is key to enabling them to initiate helpful conversations with their vaccinehesitant patients. Anger is a very understandable initial response given the context of the ongoing COVID-19 service demands, and HCWs should not feel ashamed of experiencing this. The issue is not to deny the feeling, but to recognise it and then find constructive ways to manage it. It is essential to create a gap between the feeling and what you do next. For example, walk away and discuss with a colleague before going back to talk to the patient. Alternatively, one could use skills of engagement such as motivational interviewing (MI), which would assist in dealing with an angry/ frustrated initial response. MI is an evidence-based approach based on a collaborative conversation style for strengthening patients' motivation and resolving ambivalence. ${ }^{[4]} \mathrm{MI}$ emphasises collaborative communication between HCWs and patients, characterised by non-judgementalism, neutrality, compassion and respect. ${ }^{[4]}$ It is particularly useful for addressing vaccine hesitancy given MI's focus on listening, recognising, and helping patients resolve ambivalence. ${ }^{[5]}$ Furthermore, $\mathrm{MI}$ is well suited for pressured healthcare settings given its brief nature, and research demonstrating that a range of HCWs can successfully implement it. ${ }^{[6]}$

\section{Conclusions}

Our experience has been that supportive listening and allowing vaccine-hesitant patients to express their fears and concerns, while being respectful and patient, provided them with the space to reflect and think about their decisions. Many were able to shift after a single conversation. Our role is to alleviate their fears, reassure them, and explain to them the benefits vaccination will bring to them as individuals and as a community. An important tool is empathy, rather than being dismissive of their fears. We should begin with empathy, not evidence. Evidence is not enough to reduce vaccine hesitancy. It is also important not to dismiss people's realities, as vaccine hesitancy in some communities is rooted in a long history of social exclusion and negative healthcare experiences. ${ }^{[7]}$ If we are to truly address hesitancy, we must take time to understand the reasons for an individual's hesitancy. Patients discharged from GSH who have survived admission to our high-flow units and ICUs, and particularly those who have lost someone they love due to COVID-19, can become important advocates or change makers for others in the community who are vaccine hesitant. We believe that an important step in preparation for the fourth wave is to provide HCWs with skills to facilitate behaviour change in vaccine-hesitant patients.

\section{Declaration. None.}

Acknowledgements. We thank Prof. Katherine Sorsdahl and Dr Claire van der Westhuizen for their input on motivational interviewing, Dr Bernadette Eike for her support, and the frontline COVID-19 team at GSH for their incredible service.

Author contributions. JH conceptualised and wrote the manuscript. LF and MM provided input and edited the final manuscript.

Funding. None.

Conflicts of interest. None.

1. Murphy J, Vallières F, Bentall RP, et al. Psychological characteristics associated with COVID-19 vaccine hesitancy and resistance in Ireland and the United Kingdom. Nat Commun 2021;12(1):29. https://doi. org/10.1038/s41467-020-20226-9 
2. Isaacs D. What history teaches us about vaccines and pandemics. Microbiol Aust 2020;41(4):168-171. 3. Arce JSS, Warren SS, Meriggi NF, et al. COVID-19 vaccine acceptance and hesitancy in low- and middle-income countries. Nat Med 2021;27(8):1385-1394. https://doi.org/10.1038/s41591-021$01454-\mathrm{y}$

4. Cole J, Berman S, Gardner I, McGuire K, Chen AMH. Implementation of a motivational interviewingbased decision tool to improve childhood vaccination rates: Pilot study protocol. Res Soc Admin Pharm 2021;17(3):619-624. https://doi.org/10.1016/j.sapharm.2020.04.021

5. Zolezzi M, Paravattil B, El-Gaili T. Using motivational interviewing techniques to inform decisionmaking for COVID-19 vaccination. Int J Clin Pharm 2021 (epub 2 October 2021). https://doi. org/10.1007/s11096-021-01334-y
6. Gabarda A, Butterworth SW. Using best practices to address COVID-19 vaccine hesitancy: The case for the motivational interviewing approach. Health Promot Pract 2021;22(5):611-615. https://doi. org/10.1177/15248399211016463

7. Batelaan K. 'It's not the science we distrust; it's the scientists': Reframing the anti-vaccination Batelaan K. 'Its not the science we distrust; it's the scientists': Reframing the anti-vaccination
movement within Black communities. Glob Public Health 2021 (epub 11 April 2021). https://doi.or movement within Black commun

Accepted 18 October 2021. 